\title{
Construction of Drug Intelligence Network Chain System
}

\author{
Liyan Wang ${ }^{1,2, *}$ and Xiuping Zhang ${ }^{1}$ \\ ${ }^{1}$ School of Management of Minzu University of China, Beijing, 100081 \\ ${ }^{2}$ Business school of Guilin University of Electronic Technology, Guilin, 541004 \\ *Corresponding author
}

\begin{abstract}
There are some problems in drug supply chain, such as insufficient drug research and development capacity, low modernization level of drug production management, long and inefficient drug circulation, backward informatization level of drug sales. Building drug intelligence network chain based on high-tech is an important way to improve drug service efficiency, control drug cost, improve medical service quality, and improve social and economic benefits, which helps to ensure drug safety, drug accessibility, and rational use in low population density areas.

On the basis of relevant theoretical research, this paper clarifies the concepts of drug supply chain system and drug intelligence network chain, and points out that drug intelligence network chain is a "three-flow" cooperative operation network chain based on information flow, logistics and capital flow of drug supply chain, including three-layer system structures of information sharing layer, cooperative operation layer and intelligent decision layer. Based on the current situation and existing problems of the drug supply chain, this paper designs a drug intelligent network chain scheme and constructs a business system of the drug intelligent network chain. It constructs a drug intelligent network chain coordination platform based on the "three flows" cooperative operation of information flow, logistics and capital flow to realize the construction of the drug intelligent network chain system.
\end{abstract}

Keywords-drug supply chain; intelligence network chain; drug intelligence network chain

\section{LITERATURE REVIEW}

\section{A. Drug Supply Chain}

Brennan(1998) first proposed the importance of establishing a pharmaceutical supply chain system. Then foreign scholars began to pay attention to the operation, management and informatization of the pharmaceutical supply chain, and introduced the pharmaceutical industry into the case study of the supply chain. Gatiaca $G$ et al. (2001) studied the distribution of global production capacity of pharmaceutical enterprises under uncertain conditions. L. Mockus et al. (2002) took a pharmaceutical enterprise as a case study to study the combination of production planning and daily operation scheduling. Pamela et al. (2004) introduced collaborative planning, forecasting and replenishment (CPFR) into the application of supply chain and discussed the application of this method in the pharmaceutical industry. European and American countries attach great importance to the research of safety technology in the field of drug supply chain. King Brian(2007) discussed the application of RFDI in drug supply chain. Wang Lijie (2007) thinks that the drug supply chain refers to a network consisting of four parallel sub-chains of substance chain, value chain, technology chain and information chain from the beginning to the final supply of drugs to patients. Qin Tianlei et al. (2010) believe that the core pharmaceutical manufacturing enterprises in the pharmaceutical supply chain include the whole process from the production or purchase of raw materials to the final molded products, and finally the products are delivered to the pharmaceutical consumers through the sales network. Zhang Huakang (2017) defines China's drug supply chain as a process in which drugs developed by drug research and development enterprises and processed by production enterprises are finally delivered to patients through distributors and retailers at all levels or hospitals.

\section{B. Intelligent Drug Management}

Drug sales forecasting methods based on mathematical statistics mainly include exponential smoothing model, multiple regression model, grey model, autoregressive integrated moving average model, etc. H Wang(2002) used grey model GM(1.1) to analyze and predict drug toxicity, providing scientific support for rational drug use. Wang Guangming (2004) analyzed the disadvantages of the traditional supply mode of hospital medicine in China with the supply chain management thought, put forward the JIT mode, and put forward the concrete measures to implement the JIT mode. Shen Kai (2010) established a regulatory game model for drug consumption, which provides a basis for the establishment of a drug supply chain safety management model and the use of safety management technologies.Guo Zhimin (2014) explores the construction mode of supply chain information platform by studying the problems of information system construction. Zhang Xun (2015) to establish a comprehensive and reasonable use of modern logistics theory and logistics system methods of efficient drug logistics intelligent management platfor. Hongran $\mathrm{Li}(2016)$ designed a model-free predictive control method for nonlinear systems based on polynomial regression. Cui Jiaqi (2017) studied the application of SPD logistics system in hospital drug supply chain management.

\section{DEVElopMENT OF DRUG Supply Chain}

\section{A. Definition of Concept}

- Drug Supply Chain. In the practice of drug supply chain management, there are many problems and low efficiency in drug planning and purchasing, inventory management, drug use, reverse logistics and other aspects. Deepening the reform of drug supply chain is an urgent problem to be solved in the pharmaceutical industry. The drug supply chain studied in this paper refers to the network 
chain organization in which drugs are finally delivered to consumers from drug research and development enterprises and production enterprises through circulation segments such as drug wholesale enterprises, retail enterprises and medical institutions. Covering the production, circulation, sales and consumption of drugs, the network chain is formed by connecting a series of nodes such as drug research and development providers, drug manufacturers, drug wholesalers, drug retailers, medical service institutions, drug buyers and drug users. With the help of information management system and IT technology, through the "threeflow" cooperative operation mode of information flow, logistics and capital flow, the full-chain resource integration and efficient operation of drugs from procurement, production, sales to delivery are realized.

\section{B. Drug Intelligence Network Chain.}

The drug intelligence network chain is based on the "three-flow" cooperative operation network chain of information flow, logistics and capital flow in the drug supply chain, including the three-layer architecture of information sharing layer, cooperative operation layer and intelligent decision layer.

\section{Development Status and Existing Problems of Drug Supply Chain}

\section{- Development Status of Drug Supply Chain}

With the promotion of health awareness and the wide application of high technology, high technology has provided new ways and methods for the pharmaceutical industry to provide service content and service methods. Meanwhile, consumers have undergone major changes in drug selection and drug purchase channels. The pharmaceutical industry will face a larger market with more detailed consumer groups. According to the national basic drug management system, deepening the reform of the drug supply chain and constructing a stable and sustainable drug supply chain system are the basic guarantees for drug supply. According to the statistical annual report released by the State Administration of Food and Drug Administration in 2017, by the end of November 2017, there were 4,376 production enterprises (including drug substance production enterprises and preparation production enterprises), 472,000 licensed enterprises (including 13,000 wholesale enterprises), 993,000 medical and health institutions, 30,294 hospitals (12181 public hospitals and 18113 private hospitals), 5409 retail chain enterprises, 229,000 retail chain stores and 225,000 retail pharmacies.

\section{- Problems of Drug Supply Chain}

The production, circulation, sale and consumption of drugs are important activities to safeguard and maintain people's health in modern society. Strengthening the control over the production, circulation, sale and consumption of drugs is related to the sustained and healthy development of the entire pharmaceutical industry and is the fundamental guarantee to realize the safety, effectiveness, economy and sufficiency of drugs. To improve the effectiveness of drug research and development, to reform the development pattern of "medicine to support medicine and medicine to supplement medicine" and to build an efficient drug supply chain are the requirements for the sustainable development of the entire pharmaceutical industry.At present, the development of drug supply chain cannot meet the diversified and multi-dimensional needs of consumers and the public health, and there are many problems and chaos such as repeated production, potential safety hazards, lengthy segments, shortage of drugs, vicious competition, irregular purchase and sale, etc.

Production segment. Drug production enterprises should research and produce drugs according to market demand and public health demand. The production segment of drugs is the source of drugs, is the key segment of drug quality and safety, and is directly related to the efficacy and quality safety of drugs. At present, China's pharmaceutical manufacturing enterprises are characterized by large quantity and small scale. Most pharmaceutical manufacturing enterprises have weak R\&D capability, low R\&D level, no independent intellectual property rights, and serious drug homogenization. The competition in the pharmaceutical market is fierce, and there are a large number of imitation drugs. The market stimulated by competition urges pharmaceutical manufacturers to devote most of their energy to market promotion and marketing, to excessively publicize the efficacy of drugs, and to invest insufficient in improving the research and development capabilities of drugs. As a result, it is difficult to create pharmaceutical brands and build their own core competitiveness, which is not conducive to the healthy development of the pharmaceutical market. The national drug regulatory department has made strict regulations on the whole process of drug development and production, and has vigorously promoted the GMP standard in order to do a good job of drug quality to the greatest extent and ensure drug safety and effectiveness.

Circulation segment. Drug circulation is the most numerous, difficult to manage and complex segment in the drug supply chain. Due to the large number, small scale and low efficiency of the enterprises in the circulation segment, there are too many and too long intermediate segments in the supply chain, which seriously affect the overall operation efficiency of the supply chain. In particular, there are too many levels of drug wholesale segments, and there are many levels of wholesalers, which artificially lengthen the circulation channels. There are many problems in the circulation of the drug supply chain in China, such as long segments, too many levels, the lack of extensive application of modern logistics technology and high-tech means, etc., resulting in bullwhip effect, high cost and serious waste in the drug supply chain, which seriously affects the operation efficiency of the drug supply chain and is not a large-scale, standardized, modern and standardized circulation system that meets the market requirements.

Sales segment. Drug sales segment is the final segment between drug consumers and drug users. Drug sales segment in China includes medical service institutions and retail pharmacies. The market share distribution of drug retail terminals in 2016 shows that medical institutions account for $77.5 \%$ (of which, public hospitals account for $68.4 \%$, public primary medical terminals account for $9.1 \%$ ), and retail pharmacies account for $22.5 \%$. As hospitals are allowed to operate drugs, the public medical system in our country relies heavily on drugs for its income, and there is a phenomenon of "using drugs to support medicine and using drugs to supplement medicine", which leads to major drug 
treatment ratio, over-treatment and other problems, and at the same time leads to retail chaos such as high drug prices, drug benefit delivery, and drug bribery. In recent years, with the diversification of market demand, pharmaceutical retail enterprises have developed rapidly. Due to the lack of medical talents in domestic pharmaceutical retail enterprises, it is still unable to ensure the safety and reliability of medical treatment and the effective use of drugs. At the same time, driven by interests, drug retail enterprises have the interest motivation to recommend unnecessary drugs to consumers and increase drug sales. Under China's current medical system, drug retail enterprises cannot replace the role of hospitals.

Consumption segment. The special attribute of drugs is close combination with medicine and requires high professionalism. Consumers must purchase drugs under the inspection, diagnosis and guidance of doctors or medical professionals. In most cases, consumers cannot realize their own choice of drugs. Due to the high degree of information asymmetry, the drugs selected by medical institutions or drug retail enterprises are basically drugs that consumers must accept, and consumers have little choice. In particular, because the price elasticity of demand for drugs in hospitals is very small, patients can almost only accept drugs recommended by doctors, patients seldom change their demand due to fluctuations in drug prices, and patients will only pay for their health. Due to insufficient research and development capacity and low research and development level in drug production, there are a large number of generic drugs in China's drug market, and the homogenization is serious. Consumers' purchasing behavior is easily affected by various drug marketing methods, especially some fake drug advertisements. Due to the misleading nature of false drug advertisements, it has seriously affected the safety of consumers in purchasing and using drugs, and has become an important potential safety hazard for drug sales terminals.

\section{Informatization of Drug Supply Chain}

Drug supply chain informatization has become an important reform goal of medical reform, an inevitable choice to meet market demand, and an important way to improve medical service quality, realize scientific hospital management, and improve social and economic benefits. Since the new medical reform in 2009, doctor-patient informatization has gradually been paid attention to in medical institutions. In the "12th Five-Year Plan" of 2011, the state emphasizes deepening the reform of the medical system, establishing a health information system that is both practical and can share medical information resources, and ensuring that information technology really plays a huge role in medical service management. Judging from China's policy guidance on informatization, in May 2011 the Ministry of Industry and Information Technology issued the "Medium-and Long-Term Plan for the Construction of Talents in Information Industry (2010-2020)", medical reform has invested a large amount of funds into the field of medical informatization. It is estimated that the market scale of medical informatization during the 12th Five-Year Plan period will be 80 billion, with a compound growth rate of $26 \%$. The 3521 Project will promote the rapid development of China's health informatization. IC cards, telemedicine and electronic medical records will become the focus of informatization construction during the 12th Five-Year Plan period. Due to the uneven distribution of medical resources in our country, the weak software environment and hardware conditions in most western regions, the relatively low level of informatization in medical institutions, and the lack of basic information systems such as patients, medical institutions and drugs, it is urgent to build a drug intelligence network chain system to improve and track relevant information and improve the service quality and service efficiency of medical institutions.

In recent years, the informatization of medical institutions at all levels in our country has developed rapidly. The change from paper to electronic has led to the continuous accumulation of clinical medical and management data and the formation of massive databases. However, there are still some common phenomena in the development and application of these information resources: there is a large amount of original information, but there is little processed information. Most of the isolated and scattered data are not shared jointly. Most of them are macro services, but less are micro services. Most of the data are static information, less integration of dynamic information. It is very difficult for the information department to make full use of these data to strengthen management, resulting in inefficient drug supply chain. Drug supply chain information system is in urgent need of moving from a fragmented and fragmented state to integrated integration. Through the use of information systems and the construction of information systems, drug supply chain performance can be improved and the efficiency of information systems can be better maximized.

\section{SCHEME Design OF DRUG INTELLIGENT NETWORK CHAIN SYSTEM}

\section{A. Drug Intelligence Network Chain Plan Construction Objectives}

At present, national pharmaceutical groups and regional leading pharmaceutical circulation enterprises are actively integrating resources in the upstream and downstream segments of the supply chain, promoting the integration of " logistics, information flow and capital flow" and establishing a multi-coordinated pharmaceutical supply chain system, supported by cloud computing, big data and Internet of Things technologies. Large and medium-sized pharmaceutical distribution enterprises continue to optimize and upgrade the technology of medical logistics dismantling, cold chain box turnover system, logistics whole-process visual information system, customer inquiry and service system, etc. to build an information-based intelligent supply chain. At the same time, the implementation of the "twovote system" policy has accelerated the process of flattening the pharmaceutical supply chain, and the downward shift of channel focus has become an inevitable trend. With the wisdom of the pharmaceutical supply chain and the continuous promotion of logistics standardization, it is expected that the pharmaceutical supply chain market will present an orderly competition and a steady development trend.

The establishment of information exchange channels and systems between the two sides in the strategic cooperative relationship based on computer network technology is the technical guarantee for the establishment and maintenance of the strategic cooperative relationship. 
The establishment of the computer network information system enables producers and distributors to make full use of accurate and timely market information to make correct decisions for production, sales, transportation, storage and technology development, thus ensuring the coordination and efficiency of the strategic cooperative organization and operation between producers and distributors, and achieving the goals of drug quality safety, drug accessibility and rational drug use, including the following aspects.

Based on the needs of users, perfect the humanized operation of the system. The survey found that the system users in rural areas are mostly rural doctors, with an older age structure, and most of them have been cured by the traditional manual operation mode. It is difficult to accept emerging electronic products. It is suggested to further improve the system operation in terms of simplicity and conformity with the user's operation habits. The modules of drug acceptance management, inventory management and expiration date management should be integrated into the daily purchase management module to simplify the operation process and give full play to the system functions. At the same time, increase the breadth and depth of system operation training, improve the operation level and proficiency of existing personnel through regular return visits, etc., so that the system functions can be brought into greater play.

Combining with functional requirements, perfect the system functional module design. The survey found that the top three users who have the highest demand for the system are, in turn, opening up the internal supply chain, drug payment and settlement management and real-time purchase, sale and deposit management, especially opening up the internal supply chain, which are the key problems that need to be solved urgently. Based on the demand survey and domestic and foreign experience, it is suggested to further improve the system function modules, and through the technical integration of the drug supply chain information system and the internal logistics information system of community hospitals, the drug supply chain can be opened up, and the operation of the whole supply chain can be visualized in real time. Using bar code, radio frequency identification and other technologies applied at the front end of the drug supply chain, real-time information of the drug purchasing process is collected and processed, and the whole process of drug transportation is recorded, thus completing real-time supervision of the operation of the drug supply chain and improving the operation efficiency and accuracy of the whole supply chain. It is planned to adopt a scientific in-hospital logistics operation mode to continuously optimize the entire drug supply chain so as to reduce the circulation cost in the whole chain.

Strengthen effective supervision and improve relevant policies and regulations. To strengthen the supervision of government functional departments on the drug circulation business between the drug purchaser and the drug supplier in the village clinics, mainly the purchase and sale activities in the drug purchasing and supply segments in the village clinics, so as to ensure the safety and efficiency of the drug purchasing in the village clinics. At the same time, the construction of drug supply chain management system in village clinics is huge and arduous. No matter the construction or operation of software and hardware, it cannot do without the support of the government. At present, the purchase and sale of drugs in rural areas in China still lacks a perfect legal support environment. Relevant government departments should strengthen policy support for the drug supply chain system in rural areas and improve relevant laws and regulations.

\section{B. Architecture of Drug Intelligence Network Chain}

The architecture of drug intelligence network chain includes three layers: information sharing layer, cooperative operation layer and intelligent decision layer, as shown in FIGURE I.

\section{CONSTRUCTION OF DRUG INTELLIGENCE NETWORK CHAIN SYSTEM}

\section{A. Drug Intelligence Network Chain Business System}

Drug traceability system, drug production system, drug circulation system, drug sales system, drug consumption system and drug supervision system are shown in FIGURE II.

The specific functional modules of the intelligent network chain system of the drug supply chain include the modules of drug receipt management, drug settlement, invoice management, drug inventory management and report management.

- Zero drug inventory: pharmacists in each pharmacy are responsible for confirming drug consumption in HIS system every day, and the two systems form statements according to the time node, so as to achieve "Nissin". At least once a week, the relevant personnel of the procurement department will make consumption settlement, the statements generated by the two systems will be matched, and the successfully matched data will form consumption statements, which will be submitted to supplier shangyao holding co., ltd. to issue invoices according to the quantity and amount of drugs consumed by our hospital. For drugs that do not match successfully, only the agreed price and purchase coefficient of drugs are modified in the "reconciliation failed data" to make drugs match and achieve "monthly closing".

- Drug management: The "separation of powers" approach is adopted in drug management. The list of drugs to be used is determined by the Pharmacy Administration Committee through discussion and voting. After that, the medical department issues the drug varieties to the purchasing department, which carries out the purchase. The pharmacy department supervises the receipt of drugs and the clinical pharmacists supervise the use of drugs. This will make drugs transparent from admission to hospitals to use, and prevent abuse of power for personal gains.

- Purchasing Management: The purchasing method of SPD supply chain is mainly automatic replenishment. Monitoring is set against the drugs in the basic drug list, and the lower limit of quantity is set according to certain rules. When the inventory quantity of each pharmacy on the SPD platform reaches the lower limit, the system will automatically issue a request for goods, which will be confirmed and sent by SPD staff. The drug depot divides the purchased state of drugs into 7 states: purchased, invoiced, delivered, delivered, temporarily out of stock, sent and received. 


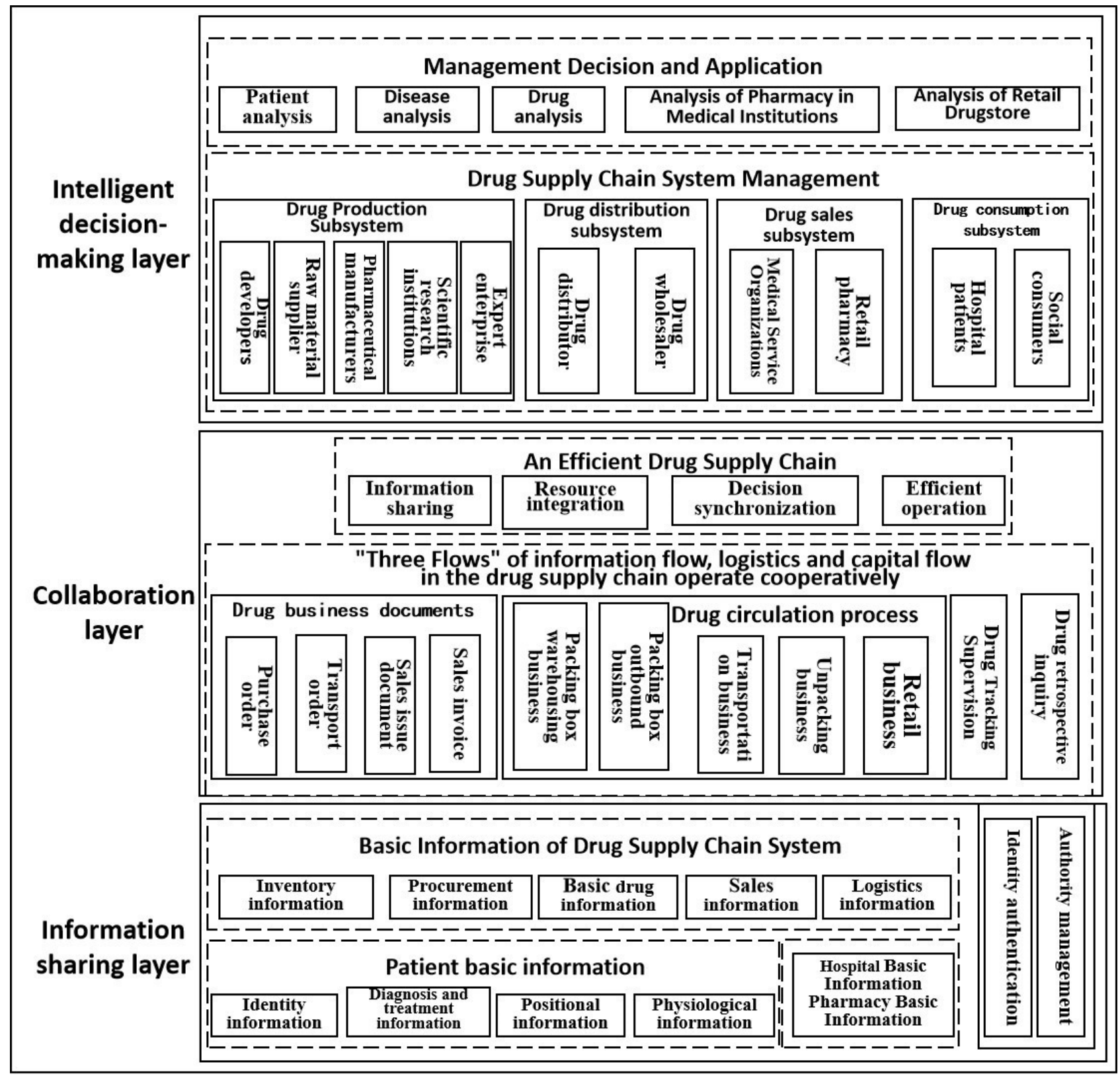

FIGURE I. ARCHITECTURE OF DRUG INTELLIGENCE NETWORK CHAIN

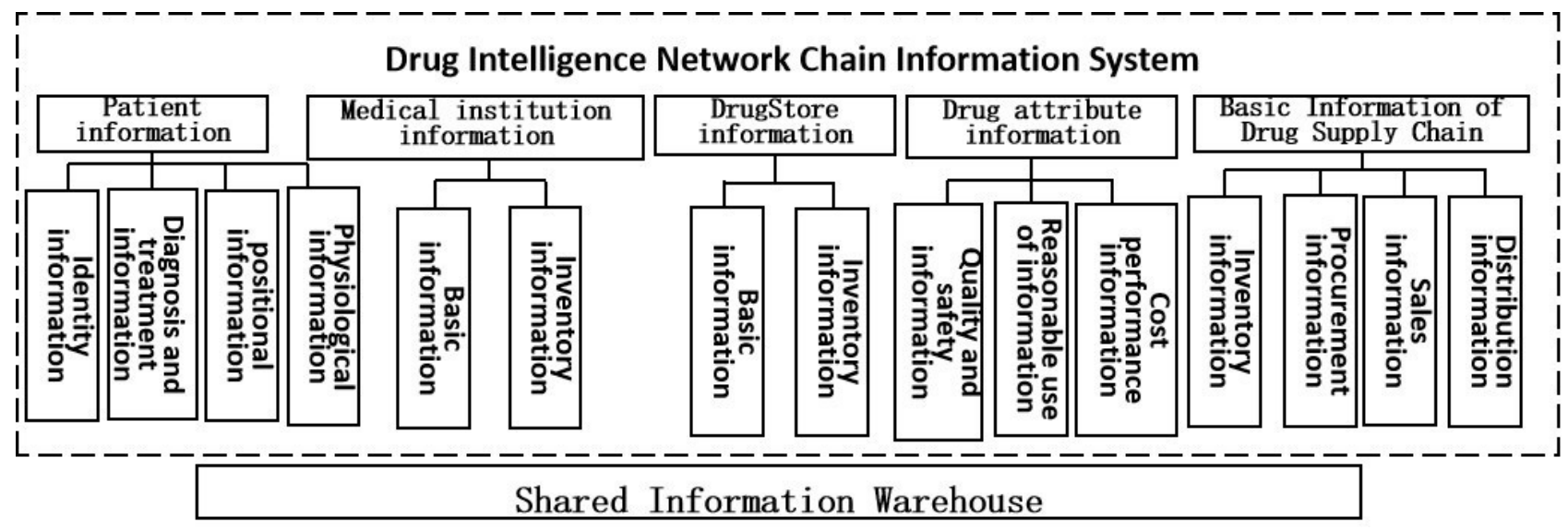

FIGURE II. DRUG INTELLIGENCE NETWORK CHAIN INFORMATION SYSTEM

Invoice management: Invoice management is divided into two parts. One is that invoices generated after the above-mentioned drugs are settled are sent to the supply chain platform in a unified way. After verification by SPD 
personnel, the relevant personnel in the procurement department of our hospital verify that the invoice details are consistent with the electronic reconciliation data and enter the payment process after confirmation. This approach avoids manual input of invoices, thus reducing the occurrence of errors, and at the same time reducing the burden on drug store staff. Second, the pharmacy of our hospital will be responsible for pharmacists' acceptance and confirmation of drugs such as refined anesthetics, which still take the same kind of drugs as the invoice. After that, the pharmacy will manually enter the invoice and record it in the account. Then the purchasing department personnel will check and confirm the invoice.

- Validity Period and Drug Maintenance: Before the 15 th of each month, SPD personnel will carry out comprehensive validity period maintenance and interrogation of drugs in various warehouses and pharmacies. Statistical validity period within 10 months of drug varieties and quantity, and fill in the monthly usage of drugs. Relevant personnel put the data production form in SPD's intranet transfer station.

- Drug distribution and use: Drug distribution: The inpatient pharmacy transfers the drugs to SPD logistics personnel, and adopts a two-way drug transfer cabinet. Pharmacists distribute the drugs from the inpatient pharmacy to each cabinet according to the ward area. Logistics personnel regularly open the cabinet door from the outside in the designated area and load the drugs from each ward area into their respective transport boxes. This not only ensures the distribution and handover of drugs, but also keeps the pharmacy clean and orderly. The SPD supply chain is transported by specialized logistics and transportation personnel, with special personnel assigned to the special area and the counter locked. Use a special medicine transportation box with a lock. After arriving at the ward, the nurse will unlock the box and count it face to face to ensure that the medicine will not be taken or touched by others during transportation. When transporting all coldchain drugs, SPD transport personnel are required to use special cold-chain boxes to ensure the quality of drugs. After pharmacists in emergency pharmacies allocate intravenous infusion drugs, they are directly and automatically transported to the infusion room by SWISSLOG transport trolley along the established track and received by emergency infusion nurses, thus avoiding the phenomenon that patients are missing or damaged when contacting infusion drugs.

- Drug use: At the end of the SPD supply chain, advanced technology management is also used, including automatic drug dispensing machines used in outpatient pharmacies and automatic drug packaging machines used in inpatient pharmacies. Outpatient service uses Swiss brand SWISSLOG automatic dispensing machine. Before taking medicine, the patient goes to the number taking machine to take the number. The machine intelligence system will divide the window and give the queue number according to the type of medicine required by the patient. Pharmacists in the hospital will be responsible for dispensing and guiding drugs as required, while pharmacy staff in SPD supply chain will be responsible for back-office allocation and replenishment of drug dispensing machines. The automatic medicine packing machine in the inpatient pharmacy uses Japanese brand Pulai and uses Japanese Tangshan medicine bag. It is also the SPD personnel who allocate the doctor's orders, which are checked again by the hospital pharmacists, and the SPD supply chain transportation personnel who send the medicines to the wards.

- Emergency Treatment: Since SPD supply chain uses information technology to distribute medicines, how to ensure the supply of medicines in case of emergencies, we have specially established an emergency plan for emergencies involving drug formulation.

\section{B. Drug Intelligence Network Chain Collaboration Platform}

Build a drug intelligence network chain collaboration platform based on the "three flows" of information flow, logistics and capital flow, as shown in FIGURE III.

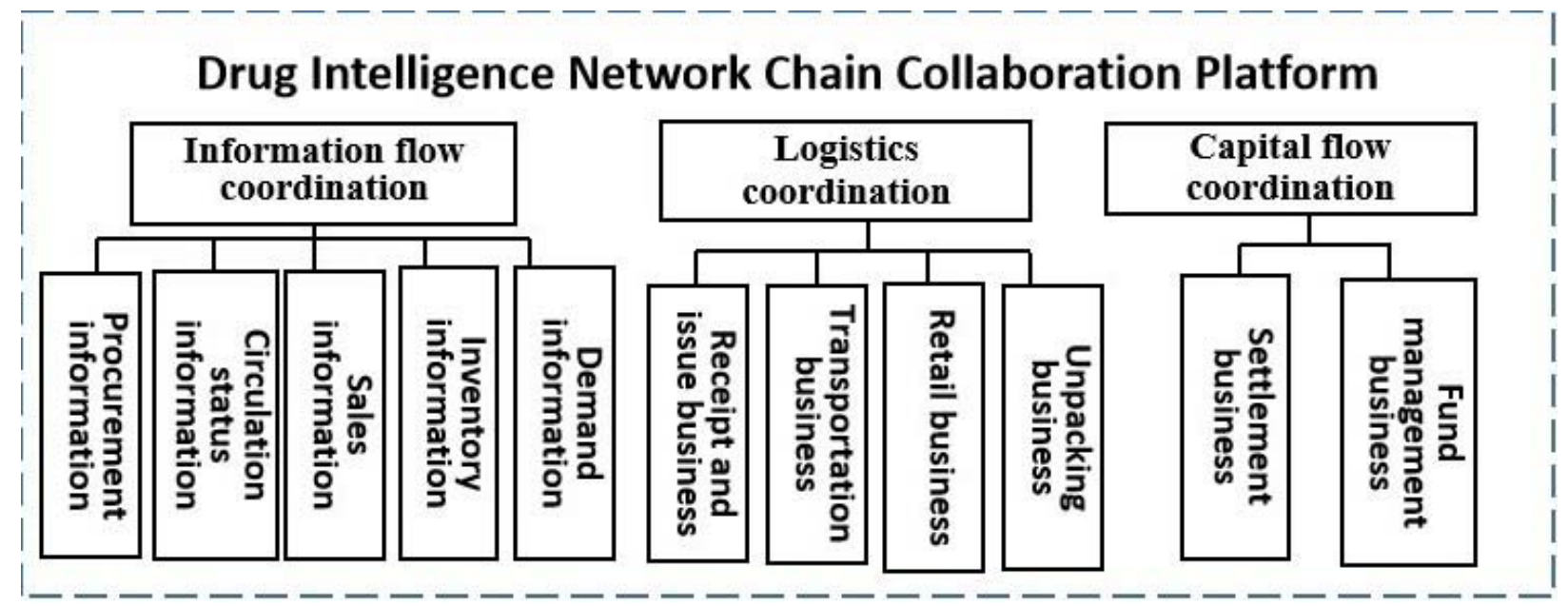

FIGURE III. DRUG INTELLIGENCE NETWORK CHAIN COORDINATION PLATFORM

There are still some problems in the drug supply chain, such as insufficient drug research and development capacity, low level of modernization of drug production and management, long and inefficient drug circulation segments, backward informatization level in drug sales segments, etc. The construction of drug intelligence network chain can 
break regional restrictions and actively introduce scientific research strength, encourage and promote drug research and development activities according to local conditions. The drug intelligence network chain establishes a strict quality assurance system to ensure the concentration of the drug industry and realize economies of scale. The cooperative operation mechanism of the drug intelligence network chain improves the overall operation capability, operation capability and management capability, realizes the standardized operation of the drug circulation market, improves the informatization level and operation efficiency of circulation segments, and improves the service quality and service efficiency of medical institutions by improving and tracking relevant information.

The establishment of a drug intelligence network chain to ensure drug quality and safety, drug accessibility, and rational use of drugs, to improve the service quality and service efficiency of medical institutions, and to enhance the health needs of residents, can deeply alleviate the social problems of "difficult to see a doctor" and "expensive to see a doctor", alleviate the doctor-patient relationship, and promote social stability and harmonious development, which are related to the well-being of the people.

\section{ACKNOWLEDGMENTS}

This research was financially supported by Basic Ability Improvement Project for Young and Middle-aged Teachers in Colleges and Universities in Guangxi of China (2018KY0183) and Independent Research Project for Doctoral Students of Minzu University of China.

\section{REFERENCES}

[1] Shi Lu Wen, 2018. Improve the drug supply guarantee system. Chinese cadres tribune.10,17-20.

[2] Pamela D., P. Romano, and A. Vinelli,2004. Managing business processes across supply networks: the role of coordination mechanisms.Journal of Purchasing and Supply Management.10,4,165-177.

[3] Liang Xu, Tang Ning, Wei Yanan,2016. Research on Drug Quality Problems in China from the Perspective of Supply Chain. Health Economics Research. 05,44-46.

[4] Sun Yongjun, 2018. Group Strategy "Coupling" Upgrade. Internet Economy,Z1,82-87.

[5] Han Zhenyan, Xia Lin, 2018. Medical care from the perspective of coupling: from mechanical combination to organic integration. Journal of Hebei University (Philosophy and Social Science .43(05),126-133.

[6] Chang Huili, Lin Jieru, Chen Qiaoyan, et al.2018. Application of SPD System in Drug Logistics Supply Chain Logistics in a Hospital. Pharmacy Today.08,569-572.

[7] Liu Y. ,2017. The dynamics of local upgrading in globalizing latecomer regions: A geographical analysis.Regional Studies.51(6),880-893. 